\title{
Patient Selection for Active Surveillance in the Multi-parametric Magnetic Resonance Imaging Era: A Step Forward in a Rapidly Evolving Field
}

\author{
Matteo Ferro, MD, $\mathrm{PhD}^{1}$, Vincenzo Mirone, $\mathrm{MD}^{2}$, Gennaro Musi, $\mathrm{MD}^{1}$, Ottavio de Cobelli, $\mathrm{MD}^{1,3}$, and \\ Massimiliano Creta, MD, $\mathbf{P h D}^{2}$ \\ ${ }^{1}$ Division of Urology, European Institute of Oncology, Milan, Italy; ${ }^{2}$ Department of Neurosciences, Sciences of \\ Reproduction, and Odontostomatology, University of Naples Federico II, Naples, Italy; ${ }^{3}$ Università degli Studi di Milano, \\ Milan, Italy
}

Active surveillance (AS) is a management option for prostate cancer $(\mathrm{PCa})$ patients that involves a close monitoring the course of the disease with the goal to intervene with curative intent if cancer progresses. ${ }^{1}$ To date, PCa patients are strongly interested in management options designed to preserve their quality of life, mainly because of the potential sexual side effects associated with available curative treatments. ${ }^{2}$ However, AS should be reserved to a highly selected group of low-risk patients, and patient selection remains a highly controversial issue. How are patients properly selected? Unfortunately, in 2018 we do not have the answer yet. The criteria most often adopted are outdated and include: Gleason score, number of positive cores, percentage of cancer involvement in every positive core, findings from digital rectal exploration, and prostate specific antigen values. ${ }^{1}$ However, they are far from being adequately accurate. Indeed, selection criteria for AS are limited by a lack of prospective, randomized, controlled trials and findings from a formal consensus meeting. ${ }^{1}$ Consequently, the risk of misclassification is still high. Multiparametric magnetic resonance imaging (mpMRI) is gaining growing interest in this clinical scenario. It represents a noninvasive tool with promising performance for selecting low-risk diseases suitable for AS. Indeed, predictive models, including both traditional clinical

(C) Society of Surgical Oncology 2018

First Received: 21 August 2018;

Published Online: 10 September 2018

M. Ferro, MD, PhD

e-mail: matteo.ferro@ieo.it parameters, and $\mathrm{mp}$-MRI findings have been reported to better discriminate between low and high-risk disease with respect to models, including only traditional parameters. However, the mp-MRI era has just begun and is in undergoing an intense and controversial evolution. In this current issue of Annals of Surgical Oncology, Yu et al. present the results of their retrospective study on $90 \mathrm{PCa}$ patients with Gleason scores $\leq 6$ potential candidates to AS who underwent radical prostatectomy between September 2009 and March 2018 designed to investigate the relationship between prebiopsy mp-MRI features, including the prostate imaging reporting and data system version 2.0 (PI-RADS v2), extraprostatic extension, and seminal vesicle invasion, and pathologic outcomes on radical prostatectomy specimen. ${ }^{3}$ The authors found that prostate-specific antigen density and PI-RADS score $>3$ were independent predictors for unfavorable pathological features. Interestingly, after replacing digital rectal examination findings with PI-RADS score groups, the model, including conventional Epstein Criteria + PI-RADS, showed superior accuracy and the discrimination power improved. ${ }^{3}$ The authors suggest that, based on the results from the present study, patients with a prebiopsy mp-MRI PI-RADS score $>3$ should be excluded from AS as its positive predictive value for predicting unfavorable disease is as high as $86.3 \% .^{3}$ The results from the present study improve the existing knowledge about the role of mp-MRI in selecting patients for AS. Indeed, the most innovative aspect is the adoption of the PI-RADS v2. This system was incorporated into radiology and urology practice in early 2015 with the goal to promote standardization and diminishing the variation in the acquisition, interpretation, and 
reporting of prostate mp-MRI examinations with respect to PI-RADS v1. ${ }^{4}$ To date, few studies have reported the use of PI-RADS v2 in evaluating potential candidates for $\mathrm{AS}^{5}{ }^{5}$ In their retrospective study, Yim et al. found that the PIRADS v2 together with tumor apparent diffusion coefficient may be a useful marker for predicting insignificant $\mathrm{PCa}$ in patients considered for AS. ${ }^{5}$ Globally, the findings from the study by $\mathrm{Yu}$ et al. are in line with published data and confirm the added value of mp-MRI in improving the accuracy of clinical models in the discrimination of $\mathrm{PCa}$ patients to candidate to AS. ${ }^{6}$ The PI-RADS v2 cutoff score of 3 in determining insignificant cancer was consistent with previous studies. ${ }^{5}$ However, as recognized by the authors, the study has several limitations that may introduce relevant biases: the retrospective single-institution design with limited sample size, the lack of data about the index tumor size on radical prostatectomy specimen that limits the possibility to compare the mp-MRI results, and pathological insignificant PCa. Moreover, as in other studies, biochemical recurrence and PCa-specific survival were not evaluated, and this may represent a limitation as unfavorable pathological characteristics do not always correlate with the prognosis. ${ }^{5}$ The PIRADS v2 system itself is still in its infancy and many limitations and ambiguities have been described. ${ }^{4}$ Moderate interobserver variability has been identified, even among expert readers and particularly for TZ evaluations. ${ }^{4}$ Moreover, PI-RADS v2 is not adequately clear about the distinction between diffusion weighted imaging scores of 3 and $>3$ for nonfocal lesions in the peripheral zone and does not sufficiently address how to classify likely nodular benign prostatic hyperplasia lesions. ${ }^{4}$ In next years, a PI-RADS v2.1 will address some of these limitations, together with other minor changes regarding data acquisition parameters and the sector map, thus potentially improving the mp-MRI interobserver variability. ${ }^{4}$ Finally, a well-designed, head-to-head comparison between PI-RADS v1 and v2 in the AS clinical scenario would be of interest.
In conclusion, we are still far from the optimum, and patient selection for AS will need future improvements. The role of PI-RADS v2 needs confirmation in further prospective, well-deigned studies. Beyond the role of PIRADS v2, the most important question remains the following: Does mp-MRI represent the future for AS? Cost issues, heterogeneity in terms of readers' experience, and concordance represent inherent limitations. Novel tools, such as serum, urinary, and tissue biomarkers, are emerging as attractive opportunities in the PCa setting, and their role as adjunctive or alternative to clinical and radiological parameters is under investigation even in the AS setting. ${ }^{7}$

\section{REFERENCES}

1. Mottet N, Bellmunt J, Bolla M, et al. EAU-ESTRO-SIOG guidelines on prostate cancer. Part 1: screening, diagnosis, and local treatment with curative intent. Eur Urol. 2017;71(4):618-29.

2. Imbimbo C, Creta M, Gacci M, et al. Patients' desire to preserve sexual activity and final decision for a nerve-sparing approach: results from the MIRROR (Multicenter Italian Report on Radical Prostatectomy Outcomes and Research) study. J Sex Med. 2011;8(5):1495-502.

3. Fan Y, Zhai L, Meng Y, et al. The contemporary Epstein Criteria with a biopsy naïve multi-parametric magnetic resonance imaging prevents a misleading into active surveillance: in PI-RADS version 2.0 era. Ann Surg Oncol. 2018. https://doi.org/10.1245/s10434-01 8-6720-2.

4. Padhani AR, Weinreb J, Rosenkrantz AB, et al. Prostate imagingreporting and data system steering committee: PI-RADS v2 status update and future directions. Eur Urol. 2018. https://doi.org/ 10.1016/j.eururo.2018.05.035.

5. Yim JH, Kim CK, Kim JH. Clinically insignificant prostate cancer suitable for active surveillance according to Prostate Cancer Research International: active surveillance criteria: utility of PIRADS v2. J Magn Reson Imaging. 2018;47(4):1072-9.

6. de Cobelli O, Terracciano D, Tagliabue E, et al. Predicting pathological features at radical prostatectomy in patients with prostate cancer eligible for active surveillance by multiparametric magnetic resonance imaging. PLoS One. 2015;10(10):e0139696.

7. Ferro M, Bruzzese $\mathrm{D}$, Perdonà $\mathrm{S}$, et al. Prostate Health Index (Phi) and Prostate Cancer Antigen 3 (PCA3) significantly improve prostate cancer detection at initial biopsy in a total PSA range of 2-10 ng/ml. PLoS One. 2013;8(7):e67687. 